\title{
Social-behavioral, clinical and anesthetic concern associated with diabetic foot amputation in a Tertiary Care Institute of North India
}

\author{
Sukhminder Jit Singh Bajwa, Smriti Anand, Amarjit Singh \\ Department of Anaesthesiology and Intensive Care, Gian Sagar Medical College and Hospital, Ram Nagar, Banur, Punjab, India
}

\section{A B S T R A C T}

Background and Aim: India has become the world capital of diabetes and its associated complications. Patients with diabetic foot lesions presenting for amputation suffer from various socio-behavioral, psychological and economic constraints, which have an impact on the care providers of those affected as well. The aim of this study was to evaluate these socio-behavioral and anesthetic concerns in those undergoing amputation so as to draw an insight to this curb the menace of diabetes. Materials and Methods: The study included I7I patients in the age group 29-78 years of either sex who underwent either amputation or debridement, followed by amputation. The clinical status was observed and duly recorded in the performa meant for the study. The socio-behavioral aspects related to diabetic foot were recorded by interview method form patients and their relatives. Results: A higher occurrence of diabetic foot morbidities was found in males (152) than in females (19) with a mean age of 52.64 years at the time of presentation in our study. Socio-behavioral factors such as low literacy levels, treatment by quacks, inadequate foot care and habit of walking barefoot, lack of physician follow-up and association of concomitant risk factors such as smoking and alcoholism and co-morbidities were important triggers for diabetic foot morbidities and subsequent amputation. These also had psychological impacts on its sufferers. Neuropathy was the main culprit accounting for $\mathbf{8 8 . 8 9 \%}$ cases, while neuropathy accounted for 1 I. $11 \%$ cases. Conclusion: Diabetic foot patients undergoing amputation require multidisciplinary management and anesthesiologist as well intensivist can play a pivotal role in psycho-social and behavioral therapy of these patients.

Key words: Amputation, diabetes mellitus, diabetic foot, socio-behavioral and psychological impacts

\section{INTRODUCTION}

Diabetic foot morbidity has seen a substantial rise throughout the globe more so in the developing nation like India. The increased prevalence of diabetic foot significantly diminishes the quality of life besides adding to the economic burden on the patient as well as on the nation. The paucity of research in this field has led to the practice of multiple therapeutic interventions.

\begin{tabular}{|l|l|}
\hline \multicolumn{2}{|c|}{ Access this article online } \\
\hline Quick Response Code: & Website: \\
\hline & www.joshd.net \\
\hline & \\
\hline
\end{tabular}

Moreover, socio-behavioral aspects of diabetic foot have not been explored or investigated as has been evident from the limited reporting in the literature. Amputation is the last resort in the therapeutic management of uncontrolled infection of the diabetic foot. However, such invasive surgical procedures are associated with various social, psychological, clinical and economic issues. The current study was undertaken to explore the various socio-behavioral, clinical and anesthetic concerns associated with amputation of the diabetic foot in patients admitted to our tertiary care institute from March 2010 to December 2013.

\section{Materials And Methods}

The present observational study was carried out in our institution in patients admitted with diabetic foot. Only

Corresponding Author: Dr. Sukhminder Jit Singh Bajwa, House No 27-A, Ratan Nagar, Tripuri, Patiala - 147001 , Punjab, India.

E-mail: sukhminder_bajwa2001@yahoo.com 
those patients were included in the study for which the amputation was carried out. A total of 171 patients at the in the age group of $29-78$ years of both gender and belonging to ASA II, and III was included in the study in whom either debridement followed by or amputation of diabetic foot was done. A detailed history and thorough systemic examination were conducted with emphasis on chronological age of diabetes and its related complications. Wherever possible, prior optimization of diabetic status was achieved before the commencement of surgical intervention with intravenous or subcutaneous insulin. Besides, other co-morbidities were also kept in consideration and careful assessment and management was done before proceeding for amputation. The clinical status was observed and duly recorded in the performa meant for the study. The socio-behavioral aspects related to diabetic foot were recorded by interview method form patients and their relatives. The questions were directed at the intellectual and educational level, awareness about the clinical aspects of diabetes, duration of diabetes, the ongoing therapeutic regimen, the adequacy of control, the other complications from diabetes, period of foot involvement, prior hospitalization for diabetes or diabetic foot, disturbance of daily routine activities as a result of foot involvement, mental preparation for amputation, awareness about risks for refusing the amputation and future plans for rehabilitation after amputation. During pre-operative examination, all these patients were also told about the important socio-behavioral and clinical aspects related to amputation of diabetic foot and adequate control of diabetes. They were specifically made aware about the need for stricter control of diabetes post amputation, need for a socio-behavioral change in daily routine life, need for strict compliance for adhering to dietary control, regular follow-up and regular screening for blood glucose.

Eleven patients had to be admitted to Intensive Care Unit (ICU) and their treatment was individualized depending upon the severity of diabetes, as well as the severity of other co-morbid illnesses. These patients and their relatives were counseled about the need for strict diabetes control during their stay in ICU as well as during the discharge from the ICU also. However, out of these eleven patients, three patients died. All the patients who were operated and discharged from hospital and ICU were told to come for a regular check-up once every month for the initial 6 months and then three monthly thereafter.

At the end of the study, data was compiled, and descriptive statistics calculations were done. All the numerical data are expressed as mean. Statistical tests could not be applied as no matching was done in the present study, and the study was mainly directed at socio-behavioral and clinical aspects related to diabetic foot.

\section{RESULTS}

The demographic profile of patients showed a higher number of male patients (152) as compared to female patients (19) presenting in our set up for diabetic foot amputations with mean age and body mass index at the time of presentation being 52.64 years and 29.36 respectively and average duration of diabetes being 7.4 years [Table 1]. 40 patients were on oral hypoglycemic agents, and 131 patients were on insulin. While assessing literacy status of the patients in study group it was found that $14.51 \%$ patients had never attended any school, $23.39 \%$ had attended up to primary school, $29.23 \% \mathrm{had}$ completed secondary schooling and $32.74 \%$ patients had attained education above secondary school. Average glycated hemoglobin levels at the time of presentation were $8.3 \%$. Eleven patients required admission in ICU because of associated co-morbidities or complication of the disease itself out of which eight patients were successfully discharged, while three patients died [Table 1].

While studying the clinical characteristics of diabetic foot ulcers neuropathy was implicated as the causative factor in $88.89 \%$ patients, while peripheral vascular disease was implicated in $11.11 \%$ patients [Table 2]. 123 patients had ambulatory difficulties, 31 patients $(18.12 \%)$ already had prior amputations of part of the foot, 48 patients (28.07\%) underwent above knee amputations, 89 (52.04\%) patients underwent below knee amputation, while 34 (19.88\%) patients had to undergo amputation at the ankle [Table 2].

On the follow-up of 168 patients, 50 were emotionally depressed while 10 patients require treatment in the form of antidepressants.

\begin{tabular}{lc}
\hline Table 1: Profile of patients with diabetic foot & \\
\hline Demographic characteristics & $\boldsymbol{n = 1 7 1}$ \\
\hline Age (in years) (mean) & 52.64 \\
Gender (male/female) & $152 / 19$ \\
Body mass index (mean) & 29.36 \\
Duration of diabetes & 7.4 years \\
Patients on oral hypoglycemic agents & 40 \\
Patients on insulin & 131 \\
Literacy levels (\%) & \\
Never attended any school & 14.51 \\
Completed primary schooling & 23.39 \\
Completed secondary schooling & 29.23 \\
Higher education (above secondary school) & 32.74 \\
Hb1Ac values (average \%) & 8.3 \\
Number of patients requiring ICU admission & 11 \\
Number of patients successfully discharged from ICU & 8 \\
Number of patients who died & 3 \\
\hline
\end{tabular}

Hb1Ac: Glycated haemoglobin; ICU: Intensive care unit 


\section{DISCUSSION}

Diabetic foot ulcers and their associated complications are one of the important culprits hampering overall qualityof-life of diabetic patients. In fact, patients with diabetic foot, related problems undergoing subsequent amputation represent a significant economic burden not only for the individual, but the society as a whole. Furthermore, the burnt of this chronic illness is borne not only by its sufferers, but also the care providers of those affected. ${ }^{[1]}$

Today India has become the world seat of diabetes with $>62$ million currently afflicted with this chronic illness, the numbers expected to rise to 79.4 million in $2030,{ }^{[2]}$ the prevalence being more so in urban than rural areas of India. ${ }^{[3]}$ This enormous increase in the diabetic population explains an alarming surge in diabetic foot lesions, a painless debilitating unwelcome surprise, which often culminates in amputation while inflicting various sociobehavioral and psychological impacts on its sufferers as observed in the present study. The need of the hour is not only to attain an adequate glycemic control in diabetics but also to allay their long-term complications, avoid disabilities and promote rehabilitation.

A higher occurrence of diabetic foot morbidities was found in males (152) than in females (19) with a mean age of 52.64 years in our study as reported by other researchers. ${ }^{[4-6]}$

Socio-behavioral aspects leading to diabetic foot amputations

Self-care activities in diabetics can go a long way in preventing complications like diabetic foot. These include behavioral adaptations in the form of dietary modifications, regular physical activity, taking regular medications (oral hypoglycemic/insulin), monitoring blood sugar levels and following foot care guidelines. ${ }^{[7]}$

While investigating 171 patients in the study group who underwent diabetic foot amputation an insight was drawn into various social and behavioral aspects of the diabetics, which escalates their risk for undergoing amputation. These included low literacy levels, treatment by quacks, inadequate foot care and habit of walking barefoot, lack of physician follow-up and association of concomitant risk factors like smoking and alcoholism and co-morbities.

Health literacy has a pivotal role in the management of diabetes and its complications. Low literacy levels results in poor comprehension of the problem and henceforth poorer compliance. ${ }^{[8]}$ Belief in alternative forms of medications being delivered by quacks poses a serious pitfall in managing and delaying complications of diabetes. In patients with neuropathy inadequate foot care, habit of walking barefoot and sleeping on the floors when they can be bitten by house rodentsall can result in diabetic foot ulcerations which are further complicated by the complete lack of podiatry in India. ${ }^{[9]}$ Lack of proper follow-up by diabetic physicians enhances the risk of diabetic foot morbidities leading to amputations. Association of concomitant risk factors such as smoking and alcoholism, as well as comorbities, are also important triggers of diabetic foot and subsequent amputations [Figure 1]. ${ }^{[6]}$

While studying the characteristics of diabetic foot lesions neuropathy was the predominant trigger $88.89 \%$, while peripheral vascular diseases accounted for meager $11.11 \%$ of diabetic foot lesions as reported by other researchers. ${ }^{[10,11]}$

Social and psychological impacts of diabetic foot amputations

Diabetic foot amputations have a negative impact on the psyche of its sufferers in the form of restriction in social activities and risk of depression. ${ }^{[12]}$ These could be further aggravated by financial constraints due to forced unemployment and feeling of being a burden on the family

\begin{tabular}{lcc}
\hline \multicolumn{3}{l}{ Table 2: Clinical characteristics of diabetic foot ulcers } \\
\hline $\begin{array}{l}\text { Clinical characteristics of } \\
\text { diabetic foot }\end{array}$ & Number of patients & Percentage \\
\hline $\begin{array}{l}\text { Neuropathy } \\
\text { Peripheral vascular disease }\end{array}$ & 152 & 88.89 \\
$\begin{array}{l}\text { Ambulatory difficulties (could not } \\
\text { walk even a smaller distance) }\end{array}$ & 19 & 11.11 \\
$\begin{array}{l}\text { Prior amputation of part of the } \\
\text { foot }\end{array}$ & 31 (digital and & 71.92 \\
$\begin{array}{l}\text { Above knee amputation } \\
\text { Below knee amputation }\end{array}$ & metatarsal amputation) & 18.12 \\
Amputation at the ankle & 88 & 28.07 \\
\hline
\end{tabular}

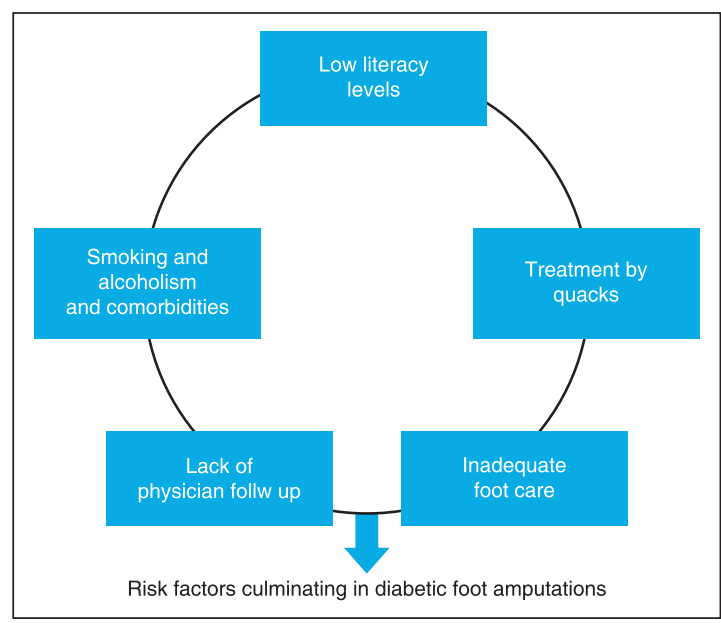

Figure 1: Risk factors culminating in diabetic foot amputations 
members. This can have detrimental effects on the care providers of those affected as well. Of the 168 patients who were followed up post amputation, 50 patients felt emotionally depressed and 10 patients, all males, required treatment in the form of antidepressants that was probably due to tremendous financial losses as they were the sole bread earners of the family [Table 3].

\section{Challenges to the anesthesiologist}

The diabetic patients especially those who are critically ill or with associated co-morbidities, presenting for amputation are always a challenge to manage for the attending anesthesiologist. Also because of their belief in alternative medications, many of these patients are on polypharmacy that further complicates their anesthetic management due to drug interactions and pathophysiological alterations. ${ }^{[13]}$

In our study, eleven patients of diabetic foot required admission in Intensive Care Unit (ICU) due to complications of diabetes mellitus and diabetic foot out of which three patients died.

\section{ROLE OF ANESTHESIOLOGIST AND INTENSIVIST}

Apart from the pivotal role in the operation theatres, the anesthesiologist can also play a key role in the psychosocial management of diabetic foot patients. This can be achieved by advocating relaxation modalities like listening to music, being tender and compassionate to their grievances, promoting yoga in the preoperative, as well as the postoperative phase. ${ }^{[14]}$ All these behavioral therapies act as stress busters as stress causes enhanced secretion of inflammatory biomarkers as well as hyperglycemia, especially in critically ill-patients. ${ }^{[15,16]}$

Diabetic foot patients landing in ICU due to associated co-morbities or complications of the disease itself pose a great challenge and responsibility to the intensivist because they have to deal not only with the critically ill patient but also the family members of those affected for whom it is difficult to accept and comprehend the need for ICU admission. Apart from the symptomatic management of the patient, the intensivist can also deliver behavioral and psychotherapy to the patient as well as the

\begin{tabular}{lc}
\hline $\begin{array}{l}\text { Table 3: Follow-up characteristics of patients after } \\
\text { amputation }\end{array}$ \\
\hline $\begin{array}{l}\text { Follow-up characteristics } \\
\text { after amputation }\end{array}$ & $\begin{array}{c}\text { Number of } \\
\text { patients }\end{array}$ \\
\hline Doing well & 108 \\
Emotionally depressed & 50 \\
Requiring antidepressants & 10 \\
\hline
\end{tabular}

family members of those affected for whom it is equally hard to come to terms to the situation. This can be done by interacting with them in their own language with empathy, breaking the myths associated with the disease, time to time information of patients condition to the relatives and explaining the grave prognosis in whom all the endeavours fail, emphasizing the need for glycemic control and regular follow-up and proper rehabilitation once they are discharged from ICU as well as emphasizing the role of Almighty as the ultimate life saver as is being practiced in our scenario. ${ }^{[14]}$

\section{CONCLUSION}

Diabetic foot lesions culminating in amputation affects its sufferers and care providers tremendously by having enormous socio-behavioral, psychological and economic impacts. With the ever increasing population of diabetics, the need of the hour is to have a multidisciplinary as well as a holistic approach in diabetes management to curb this menace. The role of anesthesiologist and intensivist today is no longer confined to the anesthetic management but by coordinating with other specialties they can play a pivotal role in managing clinical, psycho-social and behavioral aspects of diabetic foot morbidities.

\section{REFERENCES}

1. Brod M. Quality of life issues in patients with diabetes and lower extremity ulcers: Patients and care givers. Qual Life Res 1998;7:365-72.

2. Kaveeshwar SA, Cornwall J. The current state of diabetes mellitus in India. Australas Med J 2014;7:45-8.

3. Mohan V, Sandeep S, Deepa R, Shah B, Varghese C. Epidemiology of type 2 diabetes: Indian scenario. Indian J Med Res 2007;125:217-30.

4. Merza Z, Tesfaye S. The risk factors of diabetic foot ulceration. Foot 2003;13:125-9.

5. Unachukwu C, Babatunde S, Ihekwaba AE. Diabetes, hand and/ or foot ulcers: A cross-sectional hospital-based study in Port Harcourt, Nigeria. Diabetes Res Clin Pract 2007;75:148-52.

6. Shahi SK, Kumar A, Kumar S, Singh SK, Gupta SK, Singh TB. Prevalance of diabetic foot ulcer and associated risk factors in diabetic patients from North India. J Diabet Foot Complications 2012;4:83-91.

7. Goodall TA, Halford WK. Self-management of diabetes mellitus: A critical review. Health Psychol 1991;10:1-8.

8. Cavanaugh KL. Health literacy in diabetes care: Explanation, evidence and equipment. Diabetes Manag (Lond) 2011;1:191-99.

9. Pendsey $\mathrm{S}$. Clinical profile of diabetic foot in India. Int $\mathrm{J}$ Low Extrem Wounds 2010;9:180-4.

10. Viswanathan V, Kumpatla S. Pattern and causes of amputation in diabetic patients: A multicentric study from India. J Assoc Physicians India 2011;59:148-51.

11. Pendsey SP. Peripheral vascular disease: An Indian scenario. Diabetol Croat 1998;27-4:153-6.

12. Vileikyte L, Leventhal H, Gonzalez JS, Peyrot M, Rubin RR, Ulbrecht JS, et al. Diabetic peripheral neuropathy and 
depressive symptoms: The association revisited. Diabetes Care 2005;28:2378-83.

13. Bajwa SJ, Panda A. Alternative medicine and anesthesia: Implications and considerations in daily practice. Ayu 2012;33:475-80.

14. Bajwa SS, Sehgal V. Psycho-social and clinical aspects of diabeto-criticare. J Soc Health Diabetes 2013;1:70-4.

15. Manzanares W, Aramendi I. Stress hyperglycemia and its control with insulin in critically ill patients: Current evidence. Med Intensiva 2010;34:273-81.
16. Lemineur T, Deby-Dupont G, Preiser JC. Biomarkers of oxidative stress in critically ill patients: What should be measured, when and how? Curr Opin Clin Nutr Metab Care 2006;9:704-10.

How to cite this article: Bajwa SS, Anand S, Singh A. Social-behavioral, clinical and anesthetic concern associated with diabetic foot amputation in a Tertiary Care Institute of North India. J Soc Health Diabetes 2015;3:120-4.

Source of Support: Nil. Conflict of Interest: None declared. 